\title{
Acuerdos y desacuerdos entre los jefes y los subordinados sobre la admiración del liderazgo
}

\author{
Luis Felipe Llanos Reynoso* \\ Marcelo José Villarreal Coindreau**
}

Fecha de recibido: 16 de julio de 2021

Fecha de aprobado: 15 de octubre de 2021

\begin{abstract}
Para citar este artículo: Llanos Reynoso, L. F., \& Villarreal Coindreau, M. J. (2022). Acuerdos y desacuerdos entre los jefes y los subordinados sobre la admiración del liderazgo. Revista Universidad E Empresa, 24(42), 1-28. https://doi.org/10.12804/ revistas.urosario.edu.co/empresa/a.10845
\end{abstract}

\section{Resumen}

La opinión de los seguidores está tomando una mayor relevancia en la gestión del liderazgo de las organizaciones. Por ello, se buscó contrastar la percepción de los subordinados sobre si admiran a su jefe como líder, contra la opinión que tiene el propio jefe al respecto. En la muestra participaron 442 personas, 221 parejas de jefes y subordinados, de 63 empresas en México, que opinaron sobre acciones de los jefes y su liderazgo. Los resultados confirman que los subordinados tienden a admirar a sus jefes como líderes, solo si estos últimos apoyan su trabajo y confían en sus capacidades. Sin embargo, algunas opiniones de los jefes sobre su propio liderazgo, no guardan relación con lo que opinan sus subordinados. Estos hallazgos destacan la importancia de que los jefes pregunten a sus subordinados lo que opinan para que puedan fortalecer su liderazgo.

Palabras clave: liderazgo; relación jefe-líder; relación líder-seguidores; relación jefesubordinado; habilidades directivas; liderazgo transformacional; confianza; apoyo; auto eficacia.

* Doctor, Facultad de Economía y Negocios, Universidad Anáhuac, México. Correo electrónico: luis.llanos@anahuac.mx ORCID: https://orcid.org/0000-0002-8984-6314

** Doctor, Facultad de Economía y Negocios, Universidad Anáhuac, México. Correo electrónico: jose.villarreal@anahuac.mx ORCID: https://orcid.org/0000-0003-1914-8569 


\title{
Agreements and Disagreements between Bosses and Subordinates regarding the Admiration of Leadership
}

\begin{abstract}
The opinion of the followers is taking a greater relevance in the management of organizations' leadership. Therefore, it was sought to contrast the perception of subordinates about whether they admire their boss as a leader against the opinion that the boss has in this regard. The sample consisted of 442 people, 221 pairs of bosses and subordinates, from 63 companies in Mexico, who gave their opinion on the actions of the bosses and their leadership. The results confirm that subordinates tend to admire their bosses as leaders only if the latter support their work and trust their abilities. However, some opinions of bosses about their leadership are not related to what their subordinates think. These findings highlight the importance of bosses asking their subordinates what they think so they can strengthen their leadership.
\end{abstract}

Keywords: Leadership; boss-leader relationship; leader-followers relationship; boss-subordinate relationship; managerial skills; transformational leadership; confidence; support; self-efficacy.

\section{Acordos e divergências entre chefes e subordinados sobre a admiração da liderança}

\section{Resumo}

A opinião dos seguidores está ganhando cada vez mais relevância na gestão da liderança das organizações. Portanto, buscou-se contrastar a percepção dos subordinados sobre se admiram o chefe como líder com a opinião que o próprio chefe tem a esse respeito. Participaram da amostra 442 pessoas, 221 cônjuges de chefes e subordinados, de 63 empresas do México, que opinaram sobre a atuação dos chefes e de suas lideranças. Os resultados confirmam que os subordinados tendem a admirar seus chefes como líderes, apenas se estes apoiam seu trabalho e confiam em suas habilidades. No entanto, algumas opiniões dos chefes sobre sua própria liderança não estão relacionadas ao que seus subordinados pensam. Essas descobertas destacam a importância de os chefes perguntarem a seus subordinados o que eles pensam, para que possam fortalecer sua liderança.

Palavras-chave: liderança; relacionamento chefe-líder; relacionamento líder-seguidores; relação chefe-subordinado; habilidades diretivas; liderança transformacional; confiança; suporte; autoeficácia.

\section{Introducción}

\author{
El liderazgo es cada vez más difícil de conceptualizar como un fenómeno exclusivamente
} individual, por lo que han surgido nuevas teorías que lo identifican como una relación o acuerdo social, diádica, entre el líder y sus seguidores (Avery, 2011; Mayoral et al., 2018; Cetina et al., 2010). Esta idea de que el liderazgo es un fenómeno social ha impulsado una serie de estudios en Latinoamérica que abordan las interacciones de la relación líder-seguidores, en lo que se ha llamado modelos centrados en la relación. Los trabajos 
académicos latinoamericanos recomiendan profundizar el estudio de la relación líder-seguidores desde diferentes contextos, por ejemplo, Loaiza (2021) en Colombia; Carmona (2018) en Caracas; Alpaca y Ayala (2021) en Lima; Saballos et al. (2017) en Managua; y Dippel y Toledo (2004) en Valdivia.

\section{Utilidad de alcanzar un acuerdo entre jefes y subordinados sobre el liderazgo}

El acuerdo jefe-subordinado se define como el grado de similitud entre las calificaciones de un atributo (Atwater \& Yammarino, 1997). Ahora bien, al existir un acuerdo social entre jefes y subordinados sobre su liderazgo, se pueden alcanzar interacciones efectivas en las organizaciones (Tekleab et al., 2008). En particular, se vuelve más importante este acuerdo cuando el trabajo en equipo se ejerce de manera dinámica, ya que se pueden generar relaciones de alta calidad que incluyen apoyo, lealtad, respeto y confianza mutua (Tsai et al., 2017). Los líderes con un alto nivel de autoconciencia tienen una significativa comprensión de cómo sus subordinados los perciben e interpretan sus comportamientos en materia de liderazgo y pueden tener la oportunidad de influir e inspirar el logro de una visión organizacional (Ertürk et al., 2018).

\section{Ser admirado como líder}

Mientras que el liderazgo transaccional basa su influencia en la transacción definida entre jefes y subordinados (Zárate \& Matviuk, 2012), el líder transformacional basa su influencia en el carisma y la inspiración (Yukl, 2002). En este sentido, Bass y Avolio (1990) propusieron como una de las dimensiones del liderazgo transformacional, la influencia idealizada atribuida; en ella, utilizan el atributo de admiración como parte del descriptivo. Acosta et al. (2015) sostienen que esta cualidad le sirve al líder para influir en los subordinados su visión y misión, ganándose el respeto y la confianza de los demás, y anotan que "el 
líder es respetado, admirado y tiene la confianza de sus subordinados, siendo modelo de identificación e imitación para ellos" (2015, p. 43).

\section{Preguntas de investigación}

Sobre el acuerdo jefe-subordinado, se podría esperar que los jefes piensen que son admirados como líderes y que los subordinados, en efecto, admiren a sus jefes como líderes (Chiu et al., 2017; Fierro \& Villalva, 2017), sin prejuicio de la relativa horizontalidad o verticalidad de la relación o de una alta o baja distancia al poder o percepción de desigualdad, en términos de Geert Hofstede (2015). Es decir, que los jefes, en términos generales, consideren que son apreciados o altamente valorados como líderes por sus subordinados, como seguidores. Villarreal y Llanos afirman que "los superiores jerárquicos son más aceptados y admirados como líderes cuando demuestran confianza en las capacidades de sus colaboradores y contribuyen a mejorar su autoeficacia, además de apoyarlos en el trabajo" (2019, p. 19). Con estos antecedentes, se plantean las siguientes preguntas que motivan la investigación: a) ¿La admiración hacia un jefe, como líder, está relacionada con la opinión de sus subordinados con respecto a las acciones que él realiza?; b) ¿La admiración hacia un jefe, como líder, está relacionada con la opinión que él mismo tenga respecto a las acciones que él mismo realiza?; y c) ¿La admiración hacia un jefe, como líder, está relacionada con la opinión que él mismo tenga respecto de si sus subordinados lo admiran o no como líder?

Esta investigación busca ayudar a comprender mejor la relación entre las percepciones de subordinados y jefes sobre las acciones de estos que llevan a que se consideren admirados como líderes y aquéllos reporten directamente que los admiran como líderes. La novedad de este artículo va en dos sentidos, primero utilizar una muestra de trabajadores latinoamericanos, en particular de México, y segundo, ampliar el trabajo de algunos investigadores como Tsai et al. (2017) o Ertürk et al. (2018), resaltando el atributo de admiración al calificativo del líder (de la influencia idealizada atribuida), tanto en la autoevaluación del jefe como en la evaluación de los subordinados, en línea con la definición original de Bass y Avolio (1990). 
El conocimiento derivado de los análisis correspondientes permitirá que los jefes tengan una mayor conciencia de las acciones que sus subordinados valoran y la manera como pueden incidir en la admiración que sus subordinados sientan hacia ellos como líderes.

\section{Revisión de la literatura}

La primera teoría diádica del liderazgo sobre el intercambio líder-seguidor o LMx, por sus siglas en inglés, estudia la relación de las diferencias y similitudes entre el líder y sus seguidores sobre sus valores, trayectorias y características demográficas (Dansereau et al., 1975). La teoría conforma dos círculos de cercanía con el líder: uno interno (camarillas) y otro externo (periferia) (Graen \& Uhl-Bien, 1995). De igual manera, postula que la calidad de la relación es potencializada, en algunas culturas, por la competencia del seguidor (Crouch \& Yetton, 1988; Nahrgang et al., 2009; Snyder \& Bruning, 1985). Esta teoría destaca la relación entre líderes y seguidores, en contraste con el énfasis en estilos de liderazgo sugerida por otras teorías (Avery, 2011; Van Gils et al., 2010). Por otra parte, se fundamenta en diversas investigaciones sobre la "vinculación diádica vertical" (Dansereau et al., 1975); es decir, la relación entre supervisores y subordinados. Cada subordinado describe a su supervisor de una manera diferente, dependiendo de la calidad de su respectiva relación (Graen \& Uhl-Bien, 1995), y por otra, cada líder tiene una propia concepción cognitiva de autoliderazgo (Manz, 1986), dependiendo también de su respectiva relación con los subordinados.

Algunas investigaciones de la teoría LMX se enfocan en identificar, a mayor detalle, las diferencias en la relación del líder con los integrantes de su círculo interno y externo (Graen \& Uhl-Bien, 1995). Entre los resultados, se confirma que las relaciones lmx más cercanas están caracterizadas por la confianza, el respeto y sentido de obligación mutuos (Fairhurst, 1993; Graen \& Schiemann, 1978). Otras investigaciones de esta teoría se enfocan en el impacto del llamado liderazgo LMx en muy diversas variables organizacionales y cambian también a un enfoque de asociados o pares, más que de la sola relación jefe-subordinado, así como al estudio de generación de líderes (Graen \& Uhl-Bien, 1995). En estos últimos estudios se investiga cómo el entrenamiento a los supervisores para que desarrollen relaciones de 
alta calidad con todos sus subordinados influye en el desempeño y la satisfacción (Graen et al., 1982; Scandura \& Graen, 1984). Más recientemente, los investigadores interesados en el tema estudian los efectos de comparar la relación LMx personal con la que hay entre los pares y el líder (Baker \& Omilion-Hodges, 2013; Regts et al., 2018), en lo que se denomina LMX relativo (Li \& Ye, 2015) o diferenciación de LMx (Martin et al., 2018).

Los hallazgos meta-analíticos sugieren que la duración y la intensidad de la relación aumentan la coincidencia en esta percepción, medida mediante correlaciones y análisis de regresión. Además, se encuentra que el acuerdo LMx está positivamente relacionado con el compromiso organizacional y, de manera consistente con el estudio previo, con la antigüedad en la empresa (Kim et al., 2017).

La segunda teoría diádica del liderazgo es la aproximación sociocognitiva de atribución o de procesamiento de información, según la cual es fundamental la percepción de los seguidores sobre si el comportamiento y las acciones de su jefe muestran que este es un líder (Avery, 2011; Lord, 1985; Lord et al., 1984; Lord \& Maher, 1991). En línea con esta perspectiva, las propias acciones son las que definen el liderazgo (Castro \& Lupano, 2007; House et al., 2002; Kenney et al., 1994; Kenney et al, 1996). Al respecto, Van Gils et al. (2010) proponen que las variaciones en el acuerdo LMx, es decir, en las calificaciones asignadas a la calidad de una misma relación por parte del líder, respecto de las asignadas por el seguidor, se pueden explicar por las diferencias en sus percepciones de sus propios comportamientos y los comportamientos de la contraparte, o bien, por diferencias en sus respectivas teorías implícitas, tanto del liderazgo como del seguimiento; es decir, lo que se espera es que uno y otro contribuyan a la relación o lo que se considera que caracteriza a un verdadero líder o a un auténtico seguidor.

Una tercera teoría diádica, la del liderazgo transformacional, indica que la relación entre líderes y seguidores se puede fortalecer, al igual que el desempeño, cuando el jefe promueve la confianza de sus colaboradores, tanto en él como en ellos mismos, ayudándolos a crecer y a retarse para metas mayores. El liderazgo transformacional se evalúa tanto mediante la propia apreciación del supervisor como desde la percepción de sus subordinados (Avolio et al., 2010; Bass et al., 1996). Este tipo de liderazgo se considera particularmente efectivo para el logro de resultados (Bass, 1999). El liderazgo transformacional es, además, sumamente influyente dentro 
de la literatura general de recursos humanos y ampliamente investigada (Avolio \& Yammarino, 2013; Michaelis, 2009).

Algunas investigaciones profundizan en los antecedentes y las consecuencias del acuerdo entre la percepción propia y la de terceros sobre el liderazgo transformacional (Sosik \& Megerian, 1999), así como en el consenso de los seguidores sobre el liderazgo de su jefe (Felfe \& Heinitz, 2010) o un sentido más amplio del mismo (Day \& Schyns, 2010; Schyns \& Day, 2010). En línea con lo anterior, Feinberg et al. (2011) encuentran que, si un supervisor ejecuta conductas compatibles con el liderazgo transformacional, es probable que exista un acuerdo entre sus seguidores respecto a que él es un verdadero líder.

Shatzer (2009) mide el acuerdo entre la autoevaluación del supervisor y la de los subordinados sobre los comportamientos de competencia, la autoconciencia y el liderazgo transformacional de los propios supervisores. Con su investigación, Shatzer encuentra que la autoconciencia es el predictor más fuerte del liderazgo transformacional y que el cuadrado de los puntajes de diferencia predice, en un menor grado, las variables de influencia idealizada y consideración individualizada, dos de las cuatro dimensiones del liderazgo transformacional.

Algunos investigadores combinan elementos de las tres teorías anteriores para estudiar el acuerdo líder-seguidor, respecto a la influencia idealizada y el apoyo individualizado (Ertürk et al., 2018). Sus hallazgos indican que el acuerdo de ambas partes en la percepción de la influencia idealizada y el apoyo individual del jefe está asociado positivamente con la percepción de los subordinados de la calidad del intercambio líder-miembro y con la apreciación del desempeño de su supervisor en jefe.

En particular, Galliani y Vianello (2012), a partir de un estudio con representantes de ventas, identificaron la admiración por el líder como la base emocional, que da lugar al estado motivacional de inspiración, como el mejor predictor para el desarrollo de competencias en materia de orientación a metas y el mejor predictor para mantener los comportamientos cívicos organizacionales. 


\subsection{Hipótesis y modelo teórico}

Ertürk et al. estudiaron las diferentes percepciones sobre el liderazgo del jefe y sus reportes directos, en particular el "grado de congruencia entre la autoevaluación de los supervisores y la evaluación de los subordinados sobre el liderazgo transformacional de aquéllos” (2018, p. 291). Ahora bien, la presente investigación busca ampliar el trabajo de Ertürk et al. (2018), bajo un contexto latinoamericano y agregando el atributo de admiración como líder, tanto en la autoevaluación del jefe como en la evaluación de los subordinados. Sobre esta base, se propone cinco hipótesis:

1. Los jefes y los subordinados tienen las mismas opiniones sobre las acciones de los jefes como líderes; es decir, sus opiniones coinciden (H1).

2. La percepción de los jefes de que son admirados como líderes puede ser pronosticada por sus opiniones respecto de sus acciones como líderes (H2).

3. La admiración de los subordinados hacia sus jefes como líderes puede ser pronosticada por las opiniones de los subordinados sobre las acciones de sus jefes como líderes (H3).

4. La admiración de los subordinados hacia sus jefes como líderes puede ser pronosticada por las opiniones de los jefes sobre sus propias acciones como líderes (H4).

5. La opinión de los jefes "Me admira como líder" puede ser pronosticada por la opinión de los subordinados "Lo admiro como líder" (H5).

De particular interés para el presente artículo son los estudios que miden el grado en que líderes y subordinados concuerdan en su percepción de la calidad de la relación que hay entre ellos, a lo que se llama el acuerdo Lmx (Sin et al., 2009). En este sentido, es clave el estudio de pares líder-subordinado, de manera que ambas personas se refieran a la misma relación.

Partiendo de la revisión de literatura, para la definición conceptual del constructo Admiración como líder, se utilizará la definición de Galliani y Vianello (2012), quienes lo identificaron como la base emocional que da lugar al estado motivacional de inspiración. 
Para la definición conceptual del constructo Acciones del liderazgo, percibidas por los subordinados, se utilizará la definición de Felfe y Heinitz (2008), quienes lo identificaron como la observación por parte de los subordinados de los comportamientos del jefe, más que en la personalidad que él tenga; y para la definición conceptual del constructo Acciones del liderazgo, percibidas por los jefes, se tomará la definición de Ertürk et al. (2018), quienes lo identificaron como las autoevaluaciones en el desempeño de su propio liderazgo.

Como variable criterio se mide el constructo Admiración como líder y como variables predictoras se miden seis acciones de liderazgo, que consideran la opinión tanto de los jefes como de los subordinados: a) Conocimiento de las habilidades e intereses, b) Apoyo en su trabajo, c) Aprovechamiento de las habilidades e intereses, d) Confianza en las capacidades, e) Mejora de la autoeficacia y f) Mejora del autoconcepto. En la figura 1, se presenta el modelo hipotético de estudio, en el que se incluyen sus acciones de liderazgo.

Rol

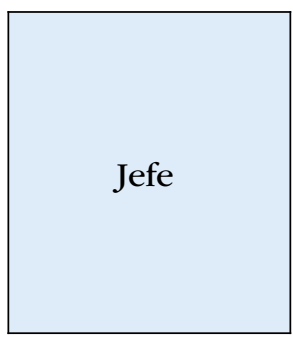

Subordinados

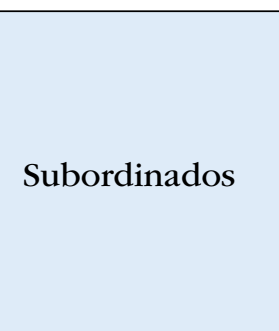

Acciones de liderazgo

\begin{tabular}{|l|}
\hline Conozco sus habilidades \\
\hline Apoyo su trabajo \\
\hline Aprovecho sus habilidades \\
\hline Confío sus capacidades \\
\hline Mejoro su autoeficacia \\
\hline Mejoro su autoconcepto \\
\hline
\end{tabular}

$\mathrm{H} 1$

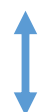

\begin{tabular}{|l|}
\hline Conoce mis habilidades \\
\hline Apoya mi trabajo \\
\hline Aprovecha mis habilidades \\
\hline Confía mis capacidades \\
\hline Mejora mi autoeficacia \\
\hline Mejora mi autoconcepto \\
\hline
\end{tabular}

Percepción de liderazgo

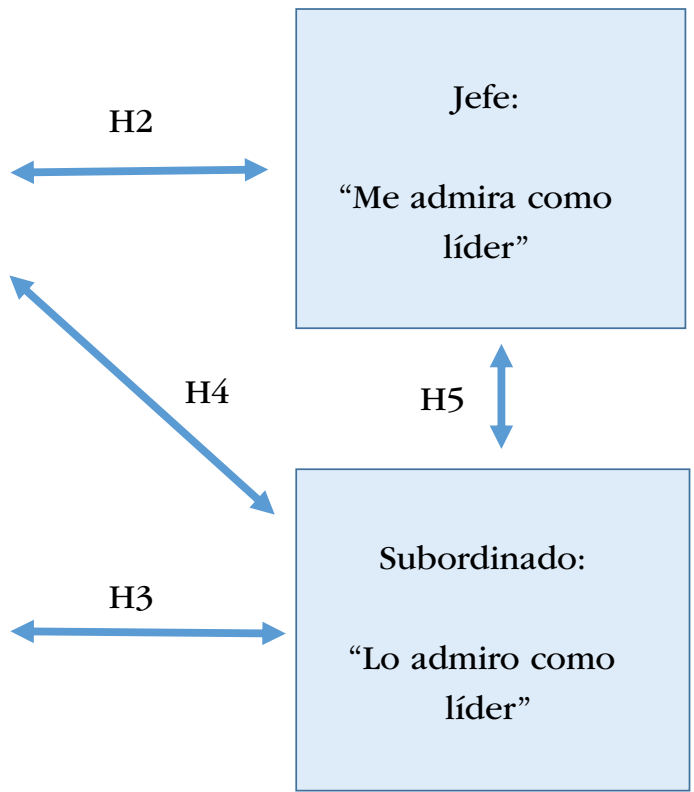

Figura 1. Modelo hipotético de variables.

Fuente: elaboración propia. 


\section{Metodología}

Para el presente estudio, se seleccionó como contexto de la investigación la ubicación de diversas diadas jefe-subordinado, de diferentes empresas en México, en las que el jefe tuviera un nivel gerencial en la empresa. La muestra para la investigación es no estadística y consta de 347 gerentes en funciones, que cursaban alguno de los programas de posgrado de una universidad privada en Huixquilucan, Estado de México, durante 2017 y 2018, en alguna de las materias de recursos humanos. En respuesta, se recibieron 329 (94.8\%) cuestionarios correspondientes al rol de jefes. Se identificaron en conjunto 63 empresas de diversas actividades donde laboraban los gerentes, siendo las principales: bancos (13.6\%), consultoría (6.8\%), seguridad (6.3\%) y educación (3.2\%). Los gerentes dirigían múltiples áreas (se identifica 48 diferentes), siendo las principales: ventas y mercadotecnia (11.3\%), producción (7.7\%), administración (5.0\%) y recursos humanos (5.0\%). A cada jefe, en promedio le reportaban directa e indirectamente 18.5 personas (con un mínimo de uno y un máximo de 400 personas).

La muestra esperada de subordinados a encuestar, con base en las estimaciones reportadas por los propios gerentes participantes, era de 870 trabajadores. Sin embargo, en respuesta a la invitación de los investigadores, se recibieron solo 234 cuestionarios, correspondientes al rol de subordinados (26.8\%). La causa principal del bajo porcentaje, en opinión de los gerentes, fue que el proceso de aplicación era totalmente voluntario, sin ninguna presión hacia ellos ni hacia sus subordinados. De las respuestas de los subordinados se identificó que el $48.0 \%$ eran hombres y el $44.2 \%$, mujeres; en tanto que, algunos participantes no declararon su sexo. El promedio de antigüedad de los subordinados en la empresa era de 5.1 años (con un mínimo de cero y un máximo de 31 años).

La investigación, utilizó la estrategia de Dansereau et al. (1984) de comparar las percepciones que existen sobre diversas acciones y pensamientos entre jefes y subordinados, por lo que solo se consideraron las diadas de la muestra en las que se pudo identificar con precisión una relación jefe-subordinado, quedando así un tamaño de muestra final de 221 diadas. 


\subsection{Instrumento de medición}

El instrumento, reportado inicialmente por Villarreal y Llanos (2019) y usado en esta investigación, se integra a partir de los elementos de la teoría del liderazgo transformacional de Bass y Avolio (1990) y de Bass et al. (2003); de los estilos apoyador y orientado a logros, propuestos por House y Mitchell (1975); el autoconcepto y la autoeficacia propuestos por Bandura (1977), así como la admiración, confianza y compromiso de los participantes con relación a su superior jerárquico de Graen y Uhl-Bien (1995) y de Polston-Murdoch (2013).

Esta combinación de variables en un solo instrumento se fundamenta en los puntos de contacto de las teorías diádicas de liderazgo, descritas en la revisión de literatura, así como en la importancia para la relación líder-seguidor, del apoyo brindado por el primero para que los segundos alcancen sus objetivos y resultados deseados (House \& Mitchell, 1975; House, 1996), lo que es posible, gracias a un autoconcepto favorable y un fuerte sentido de autoeficacia de los propios seguidores (Bandura, 1977). En el instrumento propuesto, se busca precisar esta relación de interés en la admiración, confianza y el compromiso de los seguidores hacia su líder; en este caso, de los subordinados con su superior jerárquico. En complemento a lo anterior y derivado de ello, se toma como referencia el cuestionario LMx 7 (Graen y Uhl-Bien, 1995), con su énfasis en la interacción diferenciada líder-seguidor, y el Multifactor Leadership Questionnaire, MLQ (Avolio et al., 2009; Den Hartog et al., 2011), en el que también es clave el liderazgo como relación y considera comportamientos críticos para desafiar y fortalecer, de manera individualizada, las competencias y resultados de los seguidores, para elaborar un cuestionario electrónico de 23 reactivos. Cabe destacar que el LMX 7 parte de un solo instrumento para medir la relación líder-seguidor, en tanto que el MLQ usa una versión para el líder y otra para el seguidor, en línea con la propuesta de la presente investigación.

Cada reactivo en este instrumento requiere calificarse en una escala Likert de seis puntos, donde: 1. Fuertemente en desacuerdo; 2. En desacuerdo; 3. Ligeramente en desacuerdo; 4. Ligeramente de acuerdo; 5. De acuerdo y 6. Fuertemente de acuerdo. Adicionalmente, se sigue la recomendación de Hinkin et al. (1997) que, para fines de confiabilidad en el diseño de este instrumento, cada variable debe medirse mínimo con tres reactivos. 
Ahora bien, los cuestionarios para jefes difieren solo gramaticalmente en su redacción, por razones evidentes, de los de los subordinados. Por ejemplo, el primer reactivo del cuestionario del superior jerárquico señala: 1. Pienso que me admira como líder; mientras que el primer reactivo del cuestionario del subordinado indica: 1. Lo admiro como líder. Los cuestionarios utilizados, tanto para jefes como subordinados se presentan en el anexo 1.

\subsection{Procedimiento de levantamiento de información}

Los cuestionarios electrónicos se enviaron por separado a cada uno de los participantes (tanto jefes como subordinados), siguiendo las recomendaciones de Graen (1976) de estudiar las díadas compuestas por un superior y un subalterno, a partir de intervenciones independientes. Adicionalmente, a todos los participantes se les indicó que su participación era voluntaria y que se garantizaba que ninguno de los integrantes conocería las respuestas de los demás; por lo que se les pidió que fueran lo más sinceros posible en sus respuestas. Durante el proceso, los investigadores dieron seguimiento a las respuestas de los participantes y cuando los subordinados respectivos no respondieran el cuestionario en un plazo de una semana, les enviaron solo una segunda invitación para colaborar.

\subsection{Validación del instrumento}

Se verifica la confiabilidad de cada escala, mediante coeficientes alfa de Cronbach, considerándose satisfactorios los superiores a 0.700 . En la tabla 1 se presenta los resultados por constructo para las diferentes escalas.

Tabla 1. Coeficientes del alfa de Cronbach de los constructos analizados

\begin{tabular}{lccc}
\hline \multicolumn{1}{c}{ Escalas } & Ítems & $\begin{array}{c}\text { Alfa de Cronbach de } \\
\text { cuestionarios de jefes }\end{array}$ & $\begin{array}{c}\text { Alfa de Cronbach } \\
\text { de cuestionarios } \\
\text { de subordinados }\end{array}$ \\
\hline Admiración como líder (variable criterio) & 3 & 0.821 & 0.877 \\
\hline Conocimiento de las habilidades e intereses & 5 & 0.831 & 0.904 \\
\hline Apoyo en su trabajo & 3 & 0.796 & 0.857 \\
\hline Aprovechamiento de las habilidades e intereses & 3 & 0.817 & 0.917 \\
\hline Confianza en las capacidades & 3 & 0.704 & 0.890 \\
\hline Mejora de la autoeficacia & 3 & 0.804 & 0.907 \\
\hline Mejora del autoconcepto & 3 & 0.885 & 0.944 \\
\hline
\end{tabular}

Fuente: elaboración propia. 
Los constructos de la variable criterio y las variables predictoras son confirmados para la muestra, al tener coeficientes superiores a 0.700 , tanto para el cuestionario de jefes, como para el de subordinados.

\section{Resultados}

Con la finalidad de comparar las opiniones de los jefes y las opiniones de los subordinados sobre las acciones de liderazgo de los primeros, en la tabla 2, se presenta las medias y la prueba de hipótesis de diferencia de medias de los constructos correspondientes, así como sus coeficientes de correlación de Pearson. 
Tabla 2. Prueba de hipótesis de diferencia de medias de los constructos entre sujetos

\begin{tabular}{|c|c|c|c|c|c|}
\hline Acciones de liderazgo & $\begin{array}{l}\text { Sujeto } \\
\text { evaluador }\end{array}$ & Media & SD & $\begin{array}{c}\text { Prueba } \\
\text { diferencia } \\
\text { de medias }\end{array}$ & $\begin{array}{l}\text { Coef. } \\
\text { Correl. }\end{array}$ \\
\hline \multirow{2}{*}{$\begin{array}{l}\text { Me admira como líder } \\
\text { Lo admiro como líder }\end{array}$} & Jefe & 4.841 & 0.786 & \multirow{2}{*}{0.082} & \multirow{2}{*}{0.357} \\
\hline & Subordinado & 4.989 & 0.984 & & \\
\hline \multirow{2}{*}{$\begin{array}{l}\text { Conozco sus habilidades e intereses } \\
\text { Conoce mis habilidades en intereses }\end{array}$} & Jefe & 4.909 & 0.731 & \multirow{2}{*}{0.323} & \multirow{2}{*}{0.184} \\
\hline & Subordinado & 4.828 & 0.965 & & \\
\hline \multirow{2}{*}{$\begin{array}{l}\text { Apoyo su trabajo } \\
\text { Apoya mi trabajo }\end{array}$} & Jefe & 5.101 & 0.764 & \multirow{2}{*}{0.346} & \multirow{2}{*}{0.309} \\
\hline & Subordinado & 5.021 & 1.023 & & \\
\hline \multirow{2}{*}{$\begin{array}{l}\text { Aprovecho sus habilidades } \\
\text { Aprovecha mis habilidades }\end{array}$} & Jefe & 4.947 & 0.734 & \multirow{2}{*}{0.486} & \multirow{2}{*}{0.143} \\
\hline & Subordinado & 5.006 & 1.018 & & \\
\hline \multirow{2}{*}{$\begin{array}{l}\text { Confío en sus capacidades } \\
\text { Confía en mis capacidades }\end{array}$} & Jefe & 4.988 & 0.804 & \multirow{2}{*}{0.668} & \multirow{2}{*}{0.330} \\
\hline & Subordinado & 5.027 & 1.097 & & \\
\hline \multirow{2}{*}{$\begin{array}{l}\text { Mejoro su autoeficacia } \\
\text { Mejora mi autoeficacia }\end{array}$} & Jefe & 4.929 & 0.781 & \multirow{2}{*}{0.420} & \multirow{2}{*}{0.261} \\
\hline & Subordinado & 5.002 & 1.080 & & \\
\hline \multirow{2}{*}{$\begin{array}{l}\text { Mejoro su autoconcepto } \\
\text { Mejora mi autoconcepto }\end{array}$} & Jefe & 4.795 & 0.896 & \multirow{2}{*}{0.481} & \multirow{2}{*}{0.186} \\
\hline & Subordinado & 4.864 & 1.1576 & & \\
\hline
\end{tabular}

Fuente: elaboración propia. SD = desviación estándar. Coef. Correl. = coeficiente de correlación de Pearson entre las evaluaciones de los subordinados y los jefes. Las correlaciones $r>0.130$ son significativas $(p<0.050)$.

No hay evidencia significativa $(\mathrm{p}<0.050)$ para indicar que las medias de las opiniones de los jefes y las de los subordinados son diferentes, pero tampoco hay evidencia de que sus opiniones coinciden, ya que es de destacar que las correlaciones de las evaluaciones de las acciones de liderazgo, tanto de los subordinados como de jefes, son significativas pero débiles (todas las r van de 0.143 a 0.357 con p<0.050). En particular, se destaca lo débil de las correlaciones entre Conozco sus habilidades $\mathrm{y}$ "Conoce mis habilidades" $(\mathrm{r}=0.184, \mathrm{p}=$ 0.006) y entre "Aprovecho sus habilidades" $\mathrm{y}$ "Aprovecha mis habilidades" $(\mathrm{r}=0.143, \mathrm{p}=0.034)$.

Respecto de la relación entre las variables predictoras y la variable criterio "Me admira como líder", todas calificadas por los jefes, utilizando un análisis por pasos stepwise, resulta ser significativa: $R^{2}$ ajustada $=0.560, F=71.080$ con $p<0.001$. Solo cuatro de las seis acciones estudiadas tienen coeficientes significativos ( $\mathrm{p}<0.050)$. El coeficiente significativo más representativo es "Apoyo su trabajo" [percepción de los jefes], por tener la mayor beta estandarizada. La ecuación de regresión resultante es: 
Me admira como líder $=0.416+0.326$ Apoyo su trabajo +0.216 Confío en sus capacidades + 0.191 Conozco sus habilidades + 0.151 Aprovecho sus habilidades

Por otra parte, el análisis de regresión de la variable criterio "Lo admiro como líder" utilizando el método por pasos stepwise con respecto a las variables predictoras calificadas por los subordinados, resulta ser altamente significativa: $R^{2}$ ajustada $=0.774, F=189.340$ con $\mathrm{p}<0.001$. Solo cuatro de las seis variables tienen coeficientes significativos $(\mathrm{p}<0.050)$. El coeficiente significativo más representativo es "Confía en mis capacidades" [percepción de los propios subordinados], por tener la mayor beta estandarizada. La ecuación de regresión resultante es:

Lo admiro como líder $=0.614+0.310$ Confía en mis capacidades +0.247 Apoya mi trabajo +0.163 Mejora mi autoeficacia +0.158 Conoce mis habilidades

El análisis de regresión de la variable criterio "Lo admiro como líder" utilizando el método por pasos stepwise con respecto a las variables predictoras, calificadas solo por los jefes, resulta ser significativa: $\mathrm{R}^{2}$ ajustada $=0.092, \mathrm{~F}=22.890$ con $\mathrm{p}<0.001$. Solo una de las seis variables tiene un coeficiente significativo $(\mathrm{p}<0.050)$ y es: "Confío en sus capacidades" [percepción de los propios jefes], con $\mathrm{t}=4.780$, p<0.001. La ecuación de regresión es:

Lo admiro como líder $=3.112+0.376$ Confío en sus capacidades

Respecto de la relación entre la variable predictora "Me admira como líder" y la variable criterio "Lo admiro como líder", el análisis de regresión resulta ser significativo: $\mathrm{R}^{2}$ ajustada $=0.124, \mathrm{~F}=32.020$ con $\mathrm{p}<0.001$. La ecuación de regresión resultante es:

Lo admiro como líder $=2.832+0.446$ Me admira como líder

En la figura 2 se observa las relaciones significativas identificadas entre la percepción de liderazgo y las acciones de liderazgo incluidas en los análisis de regresión lineal múltiple. Se destaca el coeficiente $\mathrm{R}^{2}=0.774$, con lo que se reafirma que el calificativo liderazgo se puede predecir por las calificaciones de los subordinados sobre sus jefes y no por los juicios que haga el jefe sobre sus propias acciones. 
Rol

Jefe

$\mathrm{r}^{2}$ entre 0.020 y 0.127

\begin{tabular}{|l|}
\hline 1. Apoyo su trabajo \\
\hline 2. Confío sus capacidades \\
\hline 3. Conozco sus habilidades \\
\hline 4. Aprovecho sus habilidades \\
\hline
\end{tabular}
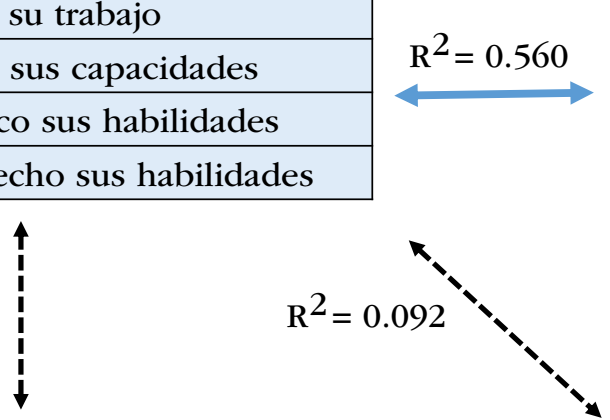

Subordinados

\begin{tabular}{|l|}
\hline 1. Confía en mis capacidades \\
\hline 2. Apoya mi trabajo \\
\hline 3. Mejora mi autoeficacia \\
\hline 4. Conoce mis habilidades \\
\hline
\end{tabular}

\section{Percepción de liderazgo}
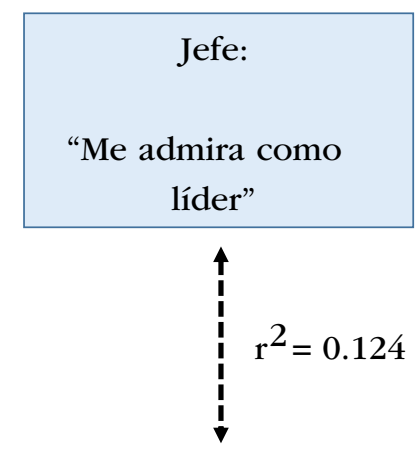

Subordinado:

"Lo admiro como

líder"

Figura 2. Diagrama ex - post de las variables significativas.

Fuente: elaboración propia. Solo se incluyen las acciones significativas de liderazgo. El orden de las variables está en función de sus betas estandarizadas.

\section{Discusión}

La evidencia sobre la hipótesis H1: Los jefes y los subordinados tienen las mismas opiniones sobre las acciones de los jefes como líderes; es decir, sus opiniones coinciden, es mixta. Por una parte, se identifica que hay una asociación positiva entre las percepciones de los subordinados con la del supervisor (entre $r=0.143$ y $r=0.357$ ), lo que concuerda con Ertürk et al. (2018); sin embargo, no existe una diferencia significativa entre ambas ( $p=0.082$ para la variable criterio y $p>0.323$ para las variables predictoras). Sin embargo, son bajos los coeficientes de correlación entre dichos pares de percepciones jefe-subordinado. Además, son bajos los coeficientes de correlación entre pares de percepciones jefe-subordinado.

Respecto de la hipótesis H2: La percepción de los jefes de que son admirados como líderes puede ser pronosticada por sus opiniones respecto de sus acciones como líderes, esta es parcialmente aceptada, ya que la variable criterio es pronosticada por una combinación de acciones que en opinión del propio jefe realiza $\left(R^{2}=0.560\right)$, destacando dentro de ellas la 
acción "Apoyo su trabajo". Este resultado es interesante a la luz de los argumentos de Van Gils et al. (2010), sobre la influencia de las teorías implícitas de liderazgo y seguimiento de los participantes en estudios diádicos, así como sobre el papel de la comunicación para disminuir el nivel de desacuerdo entre ellos. A la vez, se destaca que Shatzer (2009) reporta que la conciencia de uno mismo no presenta altas correlaciones con el acuerdo perceptual entre líderes y subordinados. Por otra parte, los resultados obtenidos en torno a esta hipótesis son consistentes con los hallazgos de Villarreal y Llanos (2019) respecto de que: la percepción de apoyo al trabajo, la confianza en las capacidades y la ayuda para incrementar la autoeficacia de los subordinados por parte del jefe, guardan relación con la percepción de este como líder.

En relación con la hipótesis H3: La admiración de los subordinados hacia sus jefes como líderes puede ser pronosticada por las opiniones de los subordinados sobre las acciones de sus jefes como líderes, es aceptada en el sentido de que la variable criterio es pronosticada con estadísticos altamente significativos $\left(R^{2}=0.774\right)$, por una combinación de acciones que en opinión del propio subordinado realiza el jefe, destacando entre ellas la acción "Confía en mis capacidades" [percepción de los propios subordinados]. Tal hallazgo coincide, desde una perspectiva diferente, con lo encontrado por Graen y Schiemann (1978) y por Fairhurst (1993) respecto a que las relaciones LMx más cercanas están caracterizadas por la confianza, entre otros elementos, y con la propuesta de Bass y sus asociados (Avolio et al., 2010; Bass, 1999; Bass et al., 1996) de que los líderes transformacionales promueven la confianza de sus subordinados en ellos mismos, ayudándolos a crecer. Esta última consideración es fortalecida porque en la predicción de la variable criterio "Lo admiro como líder" juega también un papel significativo la percepción de los colaboradores de que el jefe "Mejora mi autoeficacia".

Se destaca la diferencia que existe entre las acciones de liderazgo que sirven para pronosticar la admiración del liderazgo, ya que mientras que los jefes piensan que serán lideres si apoyan a sus subordinados (hipótesis H2), los subordinados piensan que sus jefes son líderes si tienen confianza en ellos (hipótesis H3), lo que presenta argumentos diferentes a los de Van Gils et al. (2010), quienes aportan que la perspectiva tanto de líderes como de subordinados es consistente con la descripción y predicción del liderazgo transformacional. 
Por su parte, la hipótesis H4: La admiración de los subordinados hacia sus jefes como líderes puede ser pronosticada por las opiniones de éstos últimos sobre sus propias acciones como líderes, es rechazada para predecir "Lo admiro como líder", ya que solo participa una de las seis actividades y con un pobre nivel predictivo $\left(R^{2}\right.$ ajustada $\left.=0.092\right)$.

La hipótesis H5: La opinión de los jefes "Me admira como líder" puede ser pronosticada por la opinión de los subordinados "Lo admiro como líder", también es rechazada debido a su pobre nivel predictivo $\left(\mathrm{R}^{2}\right.$ ajustada $\left.=0.124\right)$, lo que implica una falta de acuerdo entre las opiniones de los subordinados y las opiniones de los jefes, en cuanto a la calificación del liderazgo de estos últimos. Este hallazgo es consistente con resultados de diversos estudios previos reportados por Schyns y Day (2010).

En su conjunto, los resultados de la investigación indican que, si bien en esta muestra hay una baja coincidencia entre la admiración de los subordinados hacia sus jefes como líderes y la percepción de estos últimos de ser admirados como tales, así como entre tal admiración de parte de los subordinados y las acciones que los jefes perciben en sí mismos en su relación hacia los subordinados, ambos participantes concuerdan en sus teorías implícitas de liderazgo, en la importancia de la confianza y el apoyo en estos últimos para la admiración de una persona como líder, en línea con lo previsto tanto por la LMx (Graen \& Uhl-Bien, 1995) como por la teoría del liderazgo transformacional (Bass \& Avolio, 1990) y la de ruta-meta (House, 1996; House \& Mitchell, 1975).

Los hallazgos de esta investigación sugieren que, si los jefes confían y apoyan el trabajo realizado por sus subordinados, de tal modo o en tal grado que sus subordinados perciban de manera clara esas acciones, será más probable que sean vistos por estos como líderes y ellos mismos consideren que son admirados como líderes.

Como parte de esas coincidencias, el conocimiento de las capacidades del subordinado o la mejora de su autoconcepto guardaron poca relación, en ambos grupos, con la admiración hacia el jefe como líder.

En contraste, si bien tanto para los jefes como para los subordinados, el conocimiento de las habilidades de estos últimos es importante en la admiración de los primeros como 
líderes, su percepción de lo que esto implica parece ser diferente, en cuanto los jefes consideran positivo aprovechar esas habilidades, quizá como una manera de reconocimiento, los subordinados valoran más que con tal conocimiento se busque aumentar su autoeficacia.

Los hallazgos señalados en los dos párrafos previos parecen sugerir que las capacidades y el autoconcepto pudieran ser aspectos muy abstractos, remotos o lejanos a la cotidianidad de la relación jefe-subordinado, en oposición a las habilidades o, de manera paradójica, a la autoeficacia, la confianza y el apoyo, de manera más general.

Es importante explorar a mayor profundidad estas posibilidades en siguientes investigaciones.

\section{Conclusiones}

El logro de metas es sustancial para cualquier organización, por lo que al aceptar que la admiración hacia un jefe, como líder, está relacionada con las acciones que él realiza y que la admiración es el mejor predictor para el desarrollo de competencias, en materia de orientación a metas, no queda duda de que los jefes deben interesarse por obtener esta categoría por parte de los miembros de su organización y no conformarse con la opinión que ellos mismos tengan respecto a las acciones que realizan.

Estos hallazgos muestran que hay todavía un ámbito muy amplio de investigación de las coincidencias y diferencias perceptuales entre jefes y subordinados, para comprender el liderazgo desde una perspectiva relacional. Si bien hay investigaciones que muestran que las personas tienden a sentirse atraídas hacia quienes se les parecen y a ser influidas por aquellas (Felfe \& Heinitz, 2008; Felfe \& Schyns, 2010; Varela et al., 2011), la literatura sugiere que algunos rasgos de personalidad pueden influir diferencialmente en la percepción del liderazgo transformacional y aceptación de terceros (Felfe \& Schyns, 2006); por lo que será de interés analizar los posibles efectos de la propia personalidad y similitud de los seguidores con la percepción de liderazgo. 


\section{Referencias}

Acosta, J. C., Zárate, R. A., \& Garzón, M. A. (2015, julio-diciembre). Relación entre el liderazgo transformacional y la esperanza. Estudio de 100 líderes empresariales chilenos. Revista Científica "Visión de Futuro", 19(2). 38-58. https://www.redalyc.org/ pdf/3579/357941099002.pdf

Alpaca, Y. E., \& Ayala, M. (2021). Impacto del estilo de liderazgo transformacional en el índice de innovación de las empresas (Tesis de grado). Universidad Peruana de Ciencias Aplicadas, Lima. https://repositorioacademico.upc.edu.pe/handle/10757/653046

Atwater, L. E., \& Yammarino, F. J. (1997). Self-other agreement: a review and model. En G. R. Ferris (Ed.), Research in Personnel and Human Resource Management (Vol. 16, pp. 121-174). JAI Press.

Avery, G. C. (2011). Understanding leadership (3. ${ }^{a}$ ed.). Sage Publications.

Avolio, B. J., \& Yammarino, F. J. (Eds.). (2013). Transformational and charismatic leadership: The road abead 10th anniversary edition. Emerald Group Publishing Limited.

Avolio, B. J., Bass, B., \& Jung, D. I. (2010). Re-examining the components of transformational and transactional leadership using the Multifactor Leadership Questionnaire. Journal of Occupational and Organizational Psychology, 72(4), 441-462. https://doi. org/10.1348/096317999166789

Avolio, B. J., Walumbwa, F. O., \& Weber, T. J. (2009). Leadership: Current Theories, Research, and Future Directions. Annual Review of Psychology, 60, 421-449. https://doi.org/10.1146/ annurev.psych.60.110707.163621

Baker, C. R., \& Omilion-Hodges, L. M. (2013). The effect of leader-member exchange differentiation within work units on coworker exchange and organizational citizenship behaviors. Communication Research Reports, 30(4), 313-322. https://doi.org/10.1080/088240 96.2013.837387

Bandura, A. (1977). Self-efficacy: Toward a Unifying Theory of Behavioral Change. Psychological Review, 84(2), 191-215. https://doi.org/10.1037/0033-295X.84.2.191

Bass, B. (1999). Two decades of research and development in transformational leadership. European Journal of Work and Organizational Psychology, 8(1), 9-32. https://doi. org/10.1080/135943299398410

Bass, B., \& Avolio, B. (1990). The implications of transactional and transformational leadership for individual, team, and organizational development. En R. W. Woodman, \& W. A. Pasmore (Eds.), Research in Organizational Change and Development (Vol. 4, pp. 231272). Emerald Group Publishing Limited. 
Bass, B., Avolio, B. J., \& Atwater, L. E. (1996). The transformational and transactional leadership of men and women. Applied Psychology: An International Review, 45(1), 5-34. https:// doi.org/10.1111/j.1464-0597.1996.tb00847.x

Bass, B., Avolio, B. J., Jung, D. I., \& Berson, Y. (2003). Predicting Unit Performance by Assessing Transformational and Transactional Leadership. Journal of Applied Psychology, 88(2), 207218. https://doi.org/10.1037/0021-9010.88.2.207

Carmona, J. A. (2018). Relación entre el intercambio líder-miembro e intención de rotación (estudio de caso: sector académico) (Tesis de grado). Universidad Católica Andrés Bello, Caracas.

Castro, A., \& Lupano, M. L. (2007). Teorías implícitas del liderazgo y calidad de la relación entre líder y seguidor. Boletín de Psicología, (89), 7-28.

Cetina, T., Ortega, I., \& Aguilar, C. (2010). Habilidades directivas desde la percepción de los subordinados: un enfoque relacional para el estudio del liderazgo. Psicoperspectivas, 9(1), 124-137. https://doi.org/10.5027/psicoperspectivas-Vol9-Issue1-fulltext-94

Chiu, C. -Y. C., Balkundi, P., \& Weinberg, F.J. (2017, April). When managers become leaders: The role of manager network centralities, social power, and followers' perception of leadership. The Leadership Quarterly, 28(2), 334-348. https://doi.org/10.1016/j.leaqua.2016.05.004

Crouch, A., \& Yetton, P. (1988). Manager-subordinate dyads: Relationships among task and social contact, manager friendliness and subordinate performance in management groups. Organizational Behavior and Human Decision Processes, 41(1), 65-82. https://doi. org/10.1016/0749-5978(88)90047-7

Dansereau, F., Alutto, J., \& Yammarino, F. (1984). Theory testing in organizational behavior: The Varient approach. Prentice Hall.

Dansereau, F., Graen, G., \& Haga, W. J. (1975). A vertical dyad linkage approach to leadership within formal organizations: A longitudinal investigation of the role making process. Organizational Behavior and Human Performance, 13(1), 46-78. https://doi. org/10.1016/0030-5073(75)90005-7

Day, D. V., \& Schyns, B. (2010). The importance of agreement and consensus in leadership research: Introduction to the Special Issue. European Journal of Work and Organizational Psychology, 19(3), 253-258. https://doi.org/10.1080/13594320903448766

Den Hartog, D. N., Van Muijen, J. J., \& Koopman, P. L. (2011). Transactional versus transformational leadership: An analysis of the MLQ. Journal of Occupational and Organizational Psychology, 7O(1), 19-34. https://doi.org/10.1111/j.2044-8325.1997.tb00628.x 
Dippel, M., \& Toledo, M. F. (2004). Efecto del liderazgo intercambio - Líder miembro de la organización. (Tesis de grado). Facultad de ciencias económicas y administrativas, Universidad Austral de Chile, Valdivia.

Ertürk, A., Van der Broeck, H., \& Verbrigghe, J. (2018). Self-other agreement on transformational leadership and subordinates' assessment of supervisor's performance: Mediating role of leader-member exchange. Leadership \& Organization Development Journal, 39(2), 291-308. https://doi.org/10.1108/LODJ-02-2016-0048

Fairhurst, G. (1993). The leader-member exchange patterns of women leaders in industry: A discourse analysis. Communication Monographs, 60(4), 321-351. https://doi. org/10.1080/03637759309376316

Feinberg, B., Ostroff, C., \& Burke, W. W. (2011). The role of within-group agreement in understanding transformational leadership. Journal of Occupational and Organizational Psychology, 78(3), 471-488. https://doi.org/10.1348/096317905X26156

Felfe, J., \& Heinitz, K. (2008). The impact of followers' and leaders' personality and perceived similarity on followers' perceptions of transformational leadership and leader acceptance. En J. Deller (Ed.), Personality and Work (pp. 197-217). Rainer Hampp Verlag.

Felfe, J., \& Schyns, B. (2006). Personality and the perception of transformational leadership: The impact of Extraversion, Neuroticism, Personal Need for Structure, and Occupational Self-Efficacy.Journal of Applied Social Psychology, 36(3), 708-739. https://doi.org/10.1111/ j.0021-9029.2006.00026.x

Felfe, J., \& Schyns, B. (2010). Followers' personality and the perception of transformational leadership: Further evidence for the similarity hypothesis. British Journal of Management, 21(2), 393-410. https://doi.org/10.1111/j.1467-8551.2009.00649.x

Felfe, J., y Heinitz, K. (2010). The impact of consensus and agreement of leadership perceptions on commitment, organizational citizenship behaviour, and customer satisfaction. European Journal of Work and Organizational Psychology, 19(3), 279-303. https://doi. org/10.1080/13594320802708070

Fierro, I., \& Villalva, M. (2017, abril). El liderazgo Democrático: Una Aproximación Conceptual. INNOVA Research Journal, 2(4), 155-162. https://doi.org/10.33890/innova.v2.n4.2017.210

Galliani, E. M., \& Vianello, M. (2012). The emotion of admiration improves employees' goal orientations and contextual performance. International Journal of Applied Psychology, 2(4), 43-52. https://doi.org/10.5923/j.ijap.20120204.02 
Graen, G. (1976). Role-making processes within complex organizations. En M. Dunnette (Ed.), Handbook of industrial and organizational psychology (pp. 1201-1245). Rand McNally \& Co.

Graen, G., \& Schiemann, W. (1978). Leader-member agreement: A vertical dyad linkage approach. Journal of Applied Psychology, 63(2), 206-212. https://doi.org/10.1037/00219010.63.2.206

Graen, G., \& Uhl-Bien, M. (1995). Relationship-Based Approach to Leadership: Development of Leader-Member Exchange (LMx) Theory of Leadership Over 25 Years: Applying a MultiLevel Multi-Domain Perspective. The Leadership Quarterly, 6(2), 219-247. https://doi. org/10.1016/1048-9843(95)90036-5

Graen, G., Novak, M. A., \& Sommerkamp, P. (1982). The effects of leader-member exchange and job design on productivity and satisfaction: Testing a dual attachment model. Organizational Behavior and Human Performance, 30(1), 109-131. https://doi. org/10.1016/0030-5073(82)90236-7

Hinkin, T. R., Tracey, J. B., \& Enz, C. A. (1997). Scale construction: Developing reliable and valid measurement instruments. Journal of Hospitality and Tourism Research, 21(1), 100120. https://doi.org/10.1177/109634809702100108

Hofstede, G. (2015). National differences in communication styles. En D. Brzozowska, \& W. Chlopicki (Eds.), Culture's software. Communication styles (pp. 1-14). Cambridge Scholars Publishing.

House, R. J. (1996). Path-Goal Theory of Leadership: Lessons, Legacy, and a Reformulated Theory. The Leadership Quarterly, 7(3), 323-352. https://doi.org/10.1016/S1048-9843(96)90024-7

House, R. J., \& Mitchell, T. R. (1975). Path-goal theory of leadership [Technical Report]. National Technical Information Service, NTIS, U. S. Department of Commerce; University of Washington. https://apps.dtic.mil/sti/pdfs/ADA009513.pdf

House, R., Javidan, M., Hanges, P., \& Dorfman, P. (2002). Understanding cultures and implicit leadership theories across the globe: an introduction to project GLOBE. Journal of World Business, 37(1), 3-10. https://doi.org/10.1016/S1090-9516(01)00069-4

Kenney, R. A., Blascovich, J., \& Shaver, P. R. (1994). Implicit leadership theories: prototypes for new leaders. Basic and Applied Social Psychology, 15(4), 409-437. https://doi.org/10.1207/ s15324834basp1504_2

Kenney, R. A., Schwartz-Kenney, B. M., \& Blascovich, J. (1996). Implicit leadership theories: Defining leaders described as worthy of influence. Personality and Social Psychology Bulletin, 22(11), 1128-1143. https://doi.org/10.1177/01461672962211004 
Kim, B. P., Poulston, J., \& Sankaran, A. C. (2017). An examination of leader-member exchange (LMX) agreement between employees and their supervisors and its influence on work outcomes. Journal of Hospitality Marketing and Management, 26(3), 238-258. https:// doi.org/10.1080/19368623.2017.1228094

Li, B., \& Ye, M. (2015). Relative leader-member exchange: A review and agenda for future research.Journal of Service Science and Management, 8(6), 911-916. https://doi.org/10.4236/ jssm.2015.86092

Loaiza, C. T. (2021). Relación entre el género y el intercambio líder-miembro en el techo de cristal en empresas privadas de Bogotá D. C. (Tesis de grado, Facultad de psicología). Universidad Católica de Colombia. Bogotá. https://hdl.handle.net/10983/25910

Lord, R. G. (1985). An information processing approach to social perceptions, leadership perceptions and behavioral measurement on organizational settings. En B. M. Staw \& L. Cummings (Eds.), Research in Organizational Behavior (pp. 87-128). JAI Press.

Lord, R. G., \& Maher, K. J. (1991). Leadership and information processing: Linking perceptions and performance. Unwin Hyman.

Lord, R. G., Foti, R. J., \& DeVader, C. L. (1984). A test of leadership categorization theory: Internal structure, information processing, and leadership perceptions. Organizational Behavior and Human Performance, 34(3), 343-378. https://doi.org/10.1016/0030-5073(84)90043-6

Manz, C. C. (1986). Self-leadership: toward an expanded theory of self-influence processes in organizations. Academy of Management Review, 11(3), 585-600. https://doi.org/10.5465/ amr.1986.4306232

Martin, R., Thomas, G., Legood, A., \& Dello Russo, S. (2018). Leader-member exchange (LMx) differentiation and work outcomes: Conceptual clarification and critical review. Journal of Organizational Behavior, 39(2), 151-168. https://doi.org/10.1002/job.2202

Mayoral, L., Rezrazi, A., \& Gangloff, B. (2018). Si quiere que su jefe lo aprecie ¡Sonriale! Ciencias Administrativas, (15), 15-24. https://doi.org/10.24215/23143738e052

Michaelis, B. (2009). Transformational and charismatic leadership effects on performance outcomes. An analysis of linking mechanisms and boundary conditions (Tesis de grado). Ruprecht-Karls-University Heidelberg.

Nahrgang, J. D., Morgeson, F. P., \& Ilies, R. (2009). The development of leader-member exchanges: Exploring how personality and performance influence leader and member relationships over time. Organizational Behavior and Human Decision Processes, 108(2), 256-266. https://doi.org/10.1016/j.obhdp.2008.09.002

Polston-Murdoch, L. (2013). An Investigation of Path-Goal Theory, Relationship of Leadership Style, Supervisor-Related Commitment, and Gender. Emerging Leadership Journeys, 6(1), 
13-44. https://www.researchgate.net/publication/270103671_An_Investigation_of_PathGoal_Theory_Relationship_of_Leadership_Style_Supervisor-_Related_Commitment_and_ Gender

Regts, G., Molleman, E., \& Van de Brake, H. J. (2018). The impact of leader-member exchange on follower performance in light of the larger social network. Human Relations, 72(8), 1265-1291. https://doi.org/10.1177/0018726718806351

Saballos, T., Rugama, E., \& Alemán, K. V. (2017). Gestión del talento humano: El liderazgo empresarial en el éxito de la organización (Tesis de grado). Universidad Nacional Autónoma de Nicaragua, Managua. http://repositorio.unan.edu.ni/id/eprint/8726

Scandura, T. A., \& Graen, G. B. (1984). Moderating effects of initial leader-member exchange status on the effects of leadership intervention. Journal of Applied Psychology, 69(3), 428436. https://doi.org/10.1037/0021-9010.69.3.428

Schyns, B., \& Day, D. V. (2010). Critique and review of leader-member exchange theory: Issues of agreement, consensus, and excellence. European Journal of Work and Organizational Psychology, 19(1), 1-29. https://doi.org/10.1080/13594320903024922

Shatzer, R. H. (2009). Predicting transformational leadership: Self-other agreement in multisource feedback (Tesis de grado). Brigham Young University. Provo.

Sin, H.-P., Nahrgang, J. D., \& Morgeson, F. P. (2009). Understanding Why They Don't See Eye to Eye: An Examination of Leader-Member Exchange (LMx) Agreement. Journal of Applied Psychology, 94(4), 1048-1057. https://doi.org/10.1037/a0014827

Snyder, R. A., \& Bruning, N. S. (1985). Quality of vertical dyad linkages: Congruence of supervisor and subordinate competence and role stress as explanatory variables. Group and Organization Studies, 1O(1), 81-94. https://doi.org/10.1177/105960118501000105

Sosik, J. J., \& Megerian, L. E. (1999). Understanding leader emotional intelligence and performance. The role of self-other agreement on transformational leadership perceptions. Group E Organization Management, 24(3), 367-390. https://doi.org/10.1177/1059601199243006

Tekleab, A. G., Sims, H. P., Yun, S., Tesluk, P. E., \& Cox, J. (2008). Are we on the same page? Effects of self-awareness of empowering and transformational leadership. Journal of Leadership E Organizational Studies, 14(3), 185-201. https://doi.org/10.1177/1071791907311069

Tsai, C. Y., Dionne, S. D., Wang, A. C., Spain, S. M., Yammarino, F.J., \& Cheng, B. S. (2017). Effects of relational schema congruence on leader-member exchange. The Leadership Quarterly, 28(2), 268-284.

Van Gils, S., Van Quaquebeke, N., \& Van Knippenberg, D. (2010). The X-factor: On the relevance of implicit leadership and followership theories for leader-member exchange (LMX) 
agreement. European Journal of Work and Organizational Psychology, 19(3), 333-363. https://doi.org/10.1080/13594320902978458

Varela, O. E., Cater, J. J., \& Michel, N. (2011). Similarity attraction in learning contexts: An empirical study. Human Resource Development Quarterly, 22(1), 49-68. https://doi. org/10.1002/hrdq.20066

Villarreal, M. J., \& Llanos, L. F. (2019). Confianza, autoeficacia y percepción de liderazgo. Dimensión Empresarial, 17 (3), 19-36. http://dx.doi.org/10.15665/dem.v17i3.1641

Yukl, G. (2002). Leadership in organizations (5..$^{a}$ ed.). Prentice Hall.

Zárate, R., \& Matviuk, S. (2012, enero-junio). Inteligencia emocional y prácticas de liderazgo en las organizaciones colombianas. Cuadernos de administración, 28(47), 89-102. http:// www.scielo.org.co/pdf/cuadm/v28n47/v28n47a08.pdf 


\section{Anexo 1. Cuestionarios para subordinados y para jefes utilizados en la investigación}

\begin{tabular}{|c|c|c|c|}
\hline Constructo & Ítems & Cuestionario subordinado & Cuestionario jefe \\
\hline \multirow{3}{*}{$\begin{array}{l}\text { Admiración como } \\
\text { líder (variable } \\
\text { criterio) }\end{array}$} & \multirow[t]{3}{*}{3} & 1. Lo admiro como líder. & 1. Pienso que me admira como líder. \\
\hline & & 3. Confío en él y en sus decisiones. & $\begin{array}{l}\text { 3. Pienso que confía en mí y en mis decisio- } \\
\text { nes. }\end{array}$ \\
\hline & & $\begin{array}{l}\text { 23. Mi relación de trabajo con mi } \\
\text { jefe/supervisor es excelente }\end{array}$ & $\begin{array}{l}\text { 23. Mi relación de trabajo con este colabo- } \\
\text { rador es excelente }\end{array}$ \\
\hline \multirow{5}{*}{$\begin{array}{l}\text { Conocimiento de } \\
\text { las habilidades e } \\
\text { intereses }\end{array}$} & \multirow[t]{5}{*}{5} & $\begin{array}{l}\text { 2. Sé qué tan satisfecho se siente con } \\
\text { mi trabajo. }\end{array}$ & $\begin{array}{l}\text { 2. Sabe qué tan satisfecho me siento con su } \\
\text { trabajo. }\end{array}$ \\
\hline & & $\begin{array}{l}\text { 7. Comprende mis intereses labora- } \\
\text { les o profesionales. }\end{array}$ & $\begin{array}{l}\text { 7. Comprendo sus intereses y aspiraciones } \\
\text { laborales o profesionales. }\end{array}$ \\
\hline & & $\begin{array}{l}\text { 10. Conoce mis fortalezas y debilida- } \\
\text { des en mi desempeño. }\end{array}$ & $\begin{array}{l}\text { 10. Conozco las fortalezas y debilidades en } \\
\text { su desempeño. }\end{array}$ \\
\hline & & $\begin{array}{l}\text { 11. Sabe cuáles son mis aspiraciones } \\
\text { laborales o profesionales. }\end{array}$ & $\begin{array}{l}\text { 11. Sé cuáles son sus aspiraciones laborales } \\
\text { o profesionales. }\end{array}$ \\
\hline & & $\begin{array}{l}\text { 8. Conoce mis habilidades y capaci- } \\
\text { dades. }\end{array}$ & 8. Conozco sus habilidades y capacidades. \\
\hline \multirow[t]{3}{*}{$\begin{array}{l}\text { Apoyo en su } \\
\text { trabajo }\end{array}$} & \multirow[t]{3}{*}{3} & $\begin{array}{l}\text { 5. Se interesa por mis problemas y } \\
\text { necesidades. }\end{array}$ & $\begin{array}{l}\text { 5. Me intereso por sus problemas y necesi- } \\
\text { dades. }\end{array}$ \\
\hline & & $\begin{array}{l}\text { 6. Me ayuda a solucionar mis proble- } \\
\text { mas de trabajo }\end{array}$ & $\begin{array}{l}\text { 6. Lo ayudo a solucionar sus problemas de } \\
\text { trabajo }\end{array}$ \\
\hline & & 13. Reconoce mis logros. & 13. Reconozco sus logros. \\
\hline \multirow[t]{3}{*}{$\begin{array}{l}\text { Aprovechamiento } \\
\text { de las habilidades } \\
\text { e intereses }\end{array}$} & \multirow[t]{3}{*}{3} & $\begin{array}{l}\text { 18. Me asigna tareas o proyectos que } \\
\text { son compatibles con mis intereses } \\
\text { laborales o profesionales. }\end{array}$ & $\begin{array}{l}\text { 18. Le asigno tareas o proyectos que son } \\
\text { compatibles con sus intereses laborales o } \\
\text { profesionales. }\end{array}$ \\
\hline & & $\begin{array}{l}\text { 21. Me asigna tareas que son } \\
\text { acordes con mis habilidades y expe- } \\
\text { riencia. }\end{array}$ & $\begin{array}{l}\text { 21. Le asigno tareas que son acordes con } \\
\text { sus habilidades y experiencia. }\end{array}$ \\
\hline & & $\begin{array}{l}\text { 22. Me asigna tareas o proyectos que } \\
\text { me ayudan a avanzar en el logro de } \\
\text { mis aspiraciones laborales o profe- } \\
\text { sionales. }\end{array}$ & $\begin{array}{l}\text { 22. Le asigno tareas o proyectos que lo } \\
\text { ayudan a avanzar en el logro de sus aspira- } \\
\text { ciones laborales o profesionales. }\end{array}$ \\
\hline \multirow[t]{3}{*}{$\begin{array}{l}\text { Confianza en las } \\
\text { capacidades }\end{array}$} & \multirow[t]{3}{*}{3} & $\begin{array}{l}\text { 4. Me siento tratado de manera justa } \\
\text { por él. }\end{array}$ & $\begin{array}{l}\text { 4. Pienso que se siente tratado de manera } \\
\text { justa por mí. }\end{array}$ \\
\hline & & $\begin{array}{l}\text { 14. Confía en mi capacidad para } \\
\text { hacer cada vez mejor mi trabajo. }\end{array}$ & $\begin{array}{l}\text { 14. Confío en su capacidad para hacer cada } \\
\text { vez mejor su trabajo. }\end{array}$ \\
\hline & & $\begin{array}{l}\text { 20. Me ha expresado su confianza } \\
\text { de que puedo alcanzar mis anhelos } \\
\text { laborales. }\end{array}$ & $\begin{array}{l}\text { 20. Le he expresado mi confianza de que } \\
\text { puede alcanzar sus anhelos laborales. }\end{array}$ \\
\hline
\end{tabular}




\begin{tabular}{|c|c|c|c|}
\hline Constructo & Ítems & Cuestionario subordinado & Cuestionario jefe \\
\hline \multirow[t]{3}{*}{$\begin{array}{l}\text { Mejora de la auto- } \\
\text { eficacia }\end{array}$} & \multirow[t]{3}{*}{3} & $\begin{array}{l}\text { 9. Me hace sentir, de manera po- } \\
\text { sitiva, que puedo lograr mejores } \\
\text { resultados en mi trabajo de los que } \\
\text { hasta ahora he alcanzado. }\end{array}$ & $\begin{array}{l}\text { 9. Lo hago sentir, de manera positiva, que } \\
\text { puede lograr mejores resultados en su tra- } \\
\text { bajo de los que hasta ahora ha alcanzado. }\end{array}$ \\
\hline & & $\begin{array}{l}\text { 12. Con las tareas o proyectos que } \\
\text { me asigna, me reta a generar ideas } \\
\text { novedosas y nuevas soluciones. }\end{array}$ & $\begin{array}{l}\text { 12. Con las tareas o proyectos que le asigno, } \\
\text { lo reto a generar ideas novedosas y nuevas } \\
\text { soluciones. }\end{array}$ \\
\hline & & $\begin{array}{l}\text { 15. Me asigna tareas o proyectos } \\
\text { que me retan, pero que me ayudan } \\
\text { a fortalecer mis habilidades y capa- } \\
\text { cidades. }\end{array}$ & $\begin{array}{l}\text { 15. Le asigno tareas o proyectos que lo } \\
\text { retan, pero que le ayudan a fortalecer sus } \\
\text { habilidades y capacidades. }\end{array}$ \\
\hline \multirow[t]{3}{*}{$\begin{array}{l}\text { Mejora del auto- } \\
\text { concepto }\end{array}$} & \multirow[t]{3}{*}{3} & $\begin{array}{l}\text { 16. Me ha asignado tareas o pro- } \\
\text { yectos que han cambiado, positiva- } \\
\text { mente, la manera como me veo a mí } \\
\text { mismo. }\end{array}$ & $\begin{array}{l}\text { 16. Le he asignado tareas o proyectos que } \\
\text { han cambiado, positivamente, la manera } \\
\text { como se ve a sí mismo. }\end{array}$ \\
\hline & & $\begin{array}{l}\text { 17. Me asigna tareas o proyectos que } \\
\text { me retan a creer más en mí mismo. }\end{array}$ & $\begin{array}{l}\text { 17. Le asigno tareas o proyectos que lo } \\
\text { retan a creer más en sí mismo. }\end{array}$ \\
\hline & & $\begin{array}{l}\text { 19. Me asigna tareas o proyectos que } \\
\text { me retan, positivamente, a repensar } \\
\text { quién soy, "de qué estoy hecho". }\end{array}$ & $\begin{array}{l}\text { 19. Le asigno tareas o proyectos que lo re- } \\
\text { tan, positivamente, a repensar quién es, "de } \\
\text { qué está hecho". }\end{array}$ \\
\hline
\end{tabular}

Fuente: Villarreal y Llanos (2019), a partir de Bass y Avolio (1990), Bass et al. (2003), House y Mitchell (1975), Bandura (1977), Graen y Uhl-Bien (1995) y de Polston-Murdoch (2013). 